\title{
Nitric oxide dysregulation in the pathogenesis of preeclampsia among Ghanaian women
}

This article was published in the following Dove Press journal:

Integrated Blood Pressure Control

19 February 2015

Number of times this article has been viewed

\section{Kwame Adu-Bonsaffoh ${ }^{1,2}$ \\ Daniel Ansong Antwi' \\ Samuel Amenyi Obed ${ }^{3}$ \\ Ben Gyan ${ }^{4}$}

'Department of Physiology, University of Ghana Medical School, Accra, Ghana; ${ }^{2}$ Department of Obstetrics and Gynecology, Korle Bu Teaching Hospital, Accra, Ghana; ${ }^{3}$ Department of Obstetrics and Gynecology, University of Ghana Medical School, Accra, Ghana; ${ }^{4}$ Department of Immunology, Noguchi Memorial Institute for Medical Research, University of Ghana, Accra, Ghana
Correspondence: Kwame Adu-Bonsaffoh Department of Obstetrics and Gynecology, Korle Bu Teaching Hospital, PO box KB783, Korle Bu, Accra, Ghana Tel +233206300840

Email bonsaffoh@yahoo.com
Background: Preeclampsia (PE) is still a disease of theories as the exact cause remains uncertain. Widespread vascular endothelial cell dysfunction is thought to mediate the generalized vasospasm and hypertension characteristic of PE. Altered nitric oxide (NO) production has been associated with the endothelial dysfunction in the pathogenesis of PE but conflicting results have emerged from previous studies.

Objectives: To determine maternal serum levels of NO, a biomarker of endothelial function, in nonpregnant, normal pregnant, and preeclamptic women.

Materials and methods: This was a cross-sectional case-control study of 277 women comprising 75 nonpregnant, 102 normal pregnant, and 100 preeclamptic women conducted at the Korle Bu Teaching Hospital between April and June 2011. About $5 \mathrm{~mL}$ of venous blood was obtained from the participants for the various investigations after meeting the inclusion criteria and signing to a written consent. Serum levels of NO were determined by Griess reaction. The data obtained were analyzed with SPSS version 20.

Results: The study showed significantly elevated serum levels of NO in preeclamptic women $(82.45 \pm 50.31 \mu \mathrm{M})$ compared with normal pregnant $(33.12 \pm 17.81 \mu \mathrm{M})$ and nonpregnant $(16.92 \pm 11.41 \mu \mathrm{M})$ women with $P<0.001$. The alteration in maternal serum NO levels was significantly more profound in early-onset (severe) PE $(119.63 \pm 45.860 \mu \mathrm{M})$ compared to that of late-onset (mild) disease $(62.44 \pm 40.44 \mu \mathrm{M})$ with $P<0.001$, indicating a more severe vascular endothelial cell dysfunction in the early-onset disease.

Conclusion: This study has determined a profound NO upregulation in PE evidenced by significant elevation of NO metabolite levels compared to normal pregnancy. This might be due to deranged endothelial function with dysregulated production of NO to restore the persistent hypertension characteristic of PE.

Keywords: preeclampsia, endothelial dysfunction, nitric oxide, Griess reagent

\section{Introduction}

Preeclampsia (PE) is defined as a new onset of hypertension and significant proteinuria in a previously normotensive woman after the 20 th week of gestation. ${ }^{1-3}$ It is a common medical complication in pregnancy with increasing incidence globally, complicating $2 \%-8 \%$ of pregnancies worldwide. ${ }^{1,2} \mathrm{PE}$ is associated with significant maternal and perinatal morbidity and mortality. ${ }^{1,4}$ Maternal complications of PE include eclampsia (seizures), stroke, liver or renal failure, and disseminated intravascular coagulation, and fetal problems include intrauterine growth restriction and preterm birth. ${ }^{2}$ In Korle $\mathrm{Bu}$ Teaching Hospital (KBTH) where the current study was conducted, hypertensive disorders in pregnancy including PE account for over $30 \%$ of maternal deaths. ${ }^{5}$ submit your manuscript $\mid$ www.dovepress.com

Dovepress

http://dx.doi.org// 0.2/47/IBPC.S68454
Integrated Blood Pressure Control 2015:8 I-6 1 (c) (i) (2) 2015 Adu-Bonsaffoh et al. This work is published by Dove Medical Press Limited, and licensed under Creative Commons Attribution - Non Commercial (unported, v3.0) permission from Dove Medical Press Limited, provided the work is properly attributed. Permissions beyond the scope of the License are administered by Dove Medical Press Limited. Information on how to request permission may be found at: http://www.dovepress.com/permissions.php 
PE is still a disease of theories as the exact cause remains uncertain. ${ }^{3,6}$ There are no well-established strategies for primary prevention, and no consistent screening tests exist for PE. ${ }^{6}$ However, the most widely held view is that PE is a multisystem disorder with widespread vascular endothelial cell dysfunction. This vascular dysfunction is thought to mediate the characteristic generalized vasospasm manifesting as hypertension, a central feature of established PE., The major features of endothelial dysfunction include vasospasm, increased platelet aggregation, and leukocytes activation, and there is evidence that nitric oxide (NO) inhibits all these processes. $^{3}$

NO is a potent vasodilator and is thought to have a major effect on gestational vasodilation. ${ }^{2,7,8}$ Previous studies suggest that the physiologic vascular adaptation to pregnancy is accompanied by an increase in endogenous NO production and enhanced responsiveness of the vascular smooth muscle to NO. ${ }^{7,8}$ Alteration or dysregulation of NO production is thought to be associated with the pathogenesis of $\mathrm{PE}$, although conflicting results showing elevation, decrease, or no change in NO levels have emerged from previous studies. ${ }^{4,8,9}$

NO deficiency or downregulation may be associated with $\mathrm{PE}$, and NO donors have been hypothesized to prevent PE, although the evidence is contradictory. ${ }^{2}$ Accordingly, serum NO level is considered as a valuable biomarker of endothelial function, and this represents the basis of the current study. Generally, NO levels are difficult to quantify due to their relatively short half-life in the presence of oxygen and other scavenging molecules such as hemoglobin. Therefore, determination of NO levels in biological samples is usually done by measuring the nitrite and nitrate levels, which are stable products of the NO oxidation, since the concentration of these metabolites directly depends on NO production. ${ }^{10}$

In $\mathrm{PE}$, the primary disturbance appears to result from reduced uteroplacental circulation due to abnormal trophoblastic invasion of the maternal spiral arteries resulting in poor placentation. ${ }^{11}$ These spiral arteries retain their endothelial lining and the underlying smooth muscles, which make them reactive to vasoactive agents expressed by the endothelial cells. Extensive angiogenesis is vital in ensuring adequate blood supply to the developing fetus in normal pregnancy, but this angiogenic process is impaired in PE. ${ }^{11}$

Significant attempt has been made in the developed world to determine the serum level of NO in PE, ${ }^{5,7,9}$ but there are limited data in Ghana. The outcome of this work might give more clues to clinicians as to whether the pathogenesis of PE is linked to maternal serum NO levels and how such knowledge can be utilized in devising more efficacious preventive measures. The current study has aimed at determining and comparing maternal serum levels of NO in nonpregnant, normal pregnant, and preeclamptic women using the Griess reagent.

\section{Materials and methods}

This was a cross-sectional case-control study conducted at the Maternity Unit of KBTH in Accra, Ghana, between April and June 2011. KBTH is the largest tertiary hospital in Ghana with about 11,000 deliveries per year. It serves as a referral center for most of the peripheral hospitals, clinics, and maternity homes in southern Ghana.

The study participants were recruited in their third trimesters after 26 weeks of gestation. The criteria used for diagnosis of PE include a diastolic blood pressure of $90 \mathrm{mmHg}$ or higher, and/or systolic blood pressure of $140 \mathrm{mmHg}$ or higher after 20 -week gestation in a previously normotensive woman associated with significant proteinuria of $300 \mathrm{mg}$ or more in a 24-hour urine collection or $\geq 1+$ dipstick on a random urine sample. ${ }^{6}$ Early-onset PE was defined as PE that develops prior to 34 weeks of gestation, whereas late-onset PE develops at or after 34 weeks of gestation. ${ }^{12}$

The normotensive pregnant women and those diagnosed of PE were approached and evaluated for eligibility to be included in the study immediately after the diagnosis has been made. The study protocol was explained to eligible women, and those who consented were recruited into the study. The study included preeclamptic, normotensive nonpregnant, and normal pregnant women between the ages of 18 years and 42 years and without any preexisting disease. The study participants were selected using a systematic random sampling. The nonpregnant normotensive controls were recruited from the gynecology clinic of KBTH, after satisfying the inclusion criteria in the same manner. All the pregnant women included in the study must have done ultrasound scan during the first half of their pregnancies for pregnancy confirmation and accurate pregnancy dating. The cases and controls were matched for age, parity, and gestational age. Exclusion criteria consisted of patients with a history of chronic hypertension, smoking, renal disease, diabetes mellitus, chronic inflammatory diseases, urinary infection, cardiac disease, thyroid dysfunction, and infectious diseases, and women on any medical treatment other than iron and folic acid. Patients who were not able to grant informed consent and those who were unwilling to comply with the requirements of the protocol were also excluded from the study.

The study population was interviewed by means of structured questionnaire to obtain their basic demographic 
and clinical characteristics after signing an informed consent form. Other important information such as blood pressure at booking (first antenatal care) were extracted from the medical records of the patients. However, the normotensive nonpregnant women had their blood pressures measured at the time of recruitment into the study, and they were tested for proteinuria using dipsticks. Ethical clearance for the study protocol was obtained from the Ethical and Protocol Review Committee of the University of Ghana Medical School. An informed consent was obtained from all the study participants after meeting the inclusion criteria. The potential risks to the participants for taking part in the study were explained to the study participants individually. Furthermore, they were informed that failure to take part in the study would not in any way affect the normal management of their clinical conditions.

\section{Blood sampling}

About $5 \mathrm{~mL}$ of venous blood was obtained through venipuncture from all the study participants and controls after application of a tourniquet. The blood sample was centrifuged for 15 minutes at 3,000 rpm, and the serum was decanted and stored at a temperature of $-80^{\circ} \mathrm{C}$, until the laboratory assays were done. The sera were analyzed using Biosystems reagents in a semi-automated analyzer for parameters of relevance to PE such as urea, uric acid, and creatinine. Maternal serum NO was determined using the Griess reagent. ${ }^{13}$

\section{Data analysis}

The data were analyzed using SPSS version 20. Descriptive analysis was done, and the demographic, clinical, and laboratory parameters of the participants such as age, gravidity, parity, blood pressures, and NO were recorded in means \pm standard deviation (SD). The above parameters were compared between the nonpregnant, normotensive pregnant, and preeclamptic women using analysis of variance followed by a post hoc analysis using the Bonferroni's test to determine the specific differences between the parameters with significant statistical differences. The demographic, clinical, and laboratory parameters of the participants with earlyand late-onset PE were compared using the independent Student's $t$-test. A $P$-value of $<0.05$ was considered as statistically significant. The correlation between gestational age and NO levels, and between mean arterial pressure (MAP) and NO was determined using Pearson's moment correlation coefficient. MAP was calculated as diastolic blood pressure (BP) plus 1/3 (systolic BP minus diastolic BP).

\section{Results}

During the study period, 277 women were enrolled in the study comprising 100 preeclamptic, 102 normal pregnant (control group 1), and 75 nonpregnant women (control group 2). The basic demographic, clinical, and laboratory characteristics of the subjects are presented in Table 1. There were no statistically significant differences observed in the mean age and gravidity (number of pregnancies) among the nonpregnant, normotensive pregnant, and preeclamptic women. Significant differences were, however, observed in the mean parity (number of deliveries) and MAP at the time of recruitment among the normotensive nonpregnant, normotensive pregnant, and preeclamptic women. Similarly, significant differences were seen among the three groups of women regarding the mean serum levels of creatinine, urea,

Table I Demographic, clinical, and some basic laboratory data of the study participants

\begin{tabular}{|c|c|c|c|c|}
\hline Parameter & $\begin{array}{l}\text { Nonpregnant group } \\
\text { (mean } \pm \text { SD) }\end{array}$ & $\begin{array}{l}\text { Normotensive pregnant group } \\
\text { (mean } \pm \text { SD) }\end{array}$ & $\begin{array}{l}\text { Preeclamptic group } \\
\text { (mean } \pm \text { SD) }\end{array}$ & $P$-value \\
\hline Age (years) & $29.53 \pm 5.74$ & $28.11 \pm 5.58$ & $29.64 \pm 5.48$ & NS \\
\hline Gravidity & $2.44 \pm 1.54$ & $2.75 \pm 1.68$ & $2.38 \pm 1.58$ & NS \\
\hline Parity & $0.83 \pm 1.08$ & $1.26 \pm 1.30$ & $0.75 \pm 1.20^{\mathrm{a}}$ & $0.006 *$ \\
\hline Booking diastolic $\mathrm{BP}(\mathrm{mmHg})$ & NA & $65.67 \pm 8.07$ & $68.4 I \pm 7.23$ & $0.012^{*}$ \\
\hline Booking systolic BP $(\mathrm{mmHg})$ & NA & $107.95 \pm 11.69$ & || $0.26 \pm 8.9 \mid$ & NS \\
\hline Booking MAP $(\mathrm{mmHg})$ & NA & $79.76 \pm 8.24$ & $82.36 \pm 6.71$ & $0.015^{*}$ \\
\hline Diastolic BP at recruitment $(\mathrm{mmHg})$ & $71.16 \pm 8.02$ & $69.42 \pm 8.59$ & $106.28 \pm 11.70^{a, b}$ & $<0.00 I^{*}$ \\
\hline Systolic BP at recruitment $(\mathrm{mmHg})$ & $113.12 \pm 7.63$ & $111.23 \pm 9.13$ & $165.67 \pm 20.97^{\mathrm{a}, \mathrm{b}}$ & $<0.001^{*}$ \\
\hline MAP at recruitment $(\mathrm{mmHg})$ & $85.15 \pm 6.13$ & $83.36 \pm 6.99$ & $126.08 \pm 13.42^{\mathrm{a}, \mathrm{b}}$ & $<0.00 I^{*}$ \\
\hline Serum creatinine $(\mu \mathrm{mol} / \mathrm{L})$ & $94.84 \pm 13.77$ & $68.24 \pm 10.76^{b}$ & $|I 5.08 \pm 4| .44^{\mathrm{a}, \mathrm{b}}$ & $<0.00 I^{*}$ \\
\hline Serum urea $(\mathrm{mmol} / \mathrm{L})$ & $4.96 \pm 1.28$ & $2.52 \pm 1.06^{\mathrm{b}}$ & $6.55 \pm 2.90^{\mathrm{a}, \mathrm{b}}$ & $<0.00 I^{*}$ \\
\hline Serum uric acid $(\mu \mathrm{mol} / \mathrm{L})$ & $286.65 \pm 59.32$ & $224.78 \pm 75.18^{b}$ & $379.26 \pm 96.58^{a, b}$ & $<0.00 I^{*}$ \\
\hline
\end{tabular}

Notes: The results are presented as mean \pm SD. Post hoc analysis: ${ }^{a}$ significantly different from normotensive pregnant group; ${ }^{\mathrm{b}}$ significantly different from nonpregnant group. *Significant.

Abbreviations: SD, standard deviation; BP, blood pressure; MAP, mean arterial pressure; NA, not applicable; NS, nonsignificant. 
uric acid, and proteins (Table 1). The mean gestational age (weeks $\pm \mathrm{SD}$ ) at diagnosis of $\mathrm{PE}$ and recruitment of normotensive pregnant women were $33.96 \pm 2.93$ and $34.63 \pm 2.74$ $(P=0.096)$.

There was a moderate degree of negative association between the gestational age at diagnosis and NO levels in PE ( $r=0.576, P=0.005)$ with a coefficient of determination of $33.18 \%$. However, there was no statistically significant association between the MAP at diagnosis and NO levels in PE ( $r=0.164, P=0.104$ ) with a coefficient of determination of $1.08 \%$. There was a significant difference between the mean serum levels of NO in nonpregnant (16.92 $\pm 11.41 \mu \mathrm{M})$, preeclamptic $(82.45 \pm 50.31 \mu \mathrm{M})$, and normal pregnant women $(33.12 \pm 17.81 \mu \mathrm{M})$ with a $P$-value $<0.001$ (Figure 1). Post hoc analysis using the Bonferroni's method showed statistically significant difference between the three groups relative to each other (Table 1).

\section{Early-onset and late-onset PE}

Among the 100 preeclamptics studied, early-onset and late-onset PE occurred in 35 and 65 women, respectively. There was no statistically significant difference with respect to age, gravidity, parity, height, and booking systolic blood pressures between the early- and late-onset groups $(P>0.05)$. The MAP at diagnosis was significantly higher in early-onset PE $(132.36 \pm 16.91 \mathrm{mmHg})$ compared to the late-onset PE $(122.68 \pm 9.65 \mathrm{mmHg})(P<0.001)$. The mean maternal serum level of $\mathrm{NO}( \pm \mathrm{SD})$ was significantly higher in early-onset PE $(119.63 \pm 45.86 \mu \mathrm{M})$ than in late-onset disease $(62.44 \pm 40.44 \mu \mathrm{M})$ with $P<0.001$ (Figure 2).

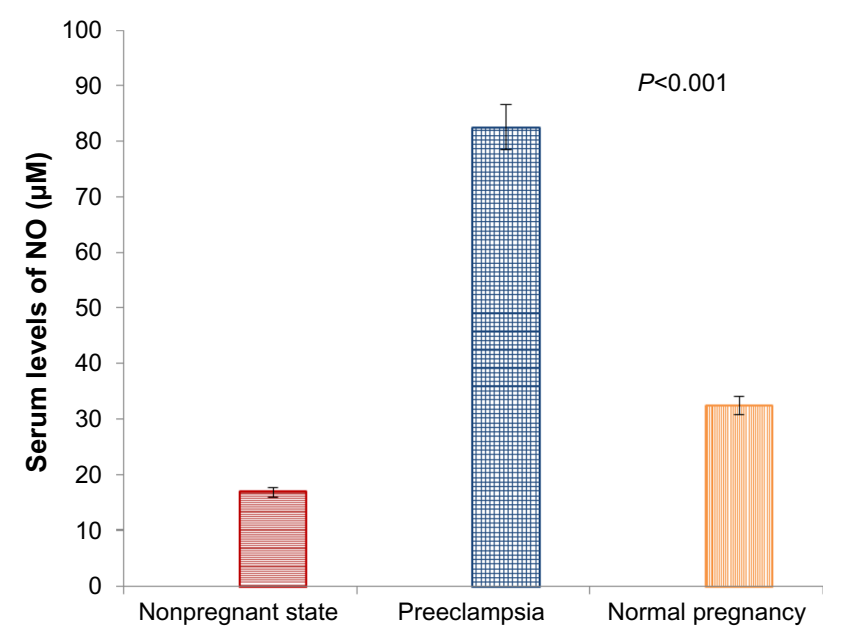

Figure I Mean serum levels of NO in normal pregnant, preeclamptic, and nonpregnant women.

Abbreviation: NO, nitric oxide.

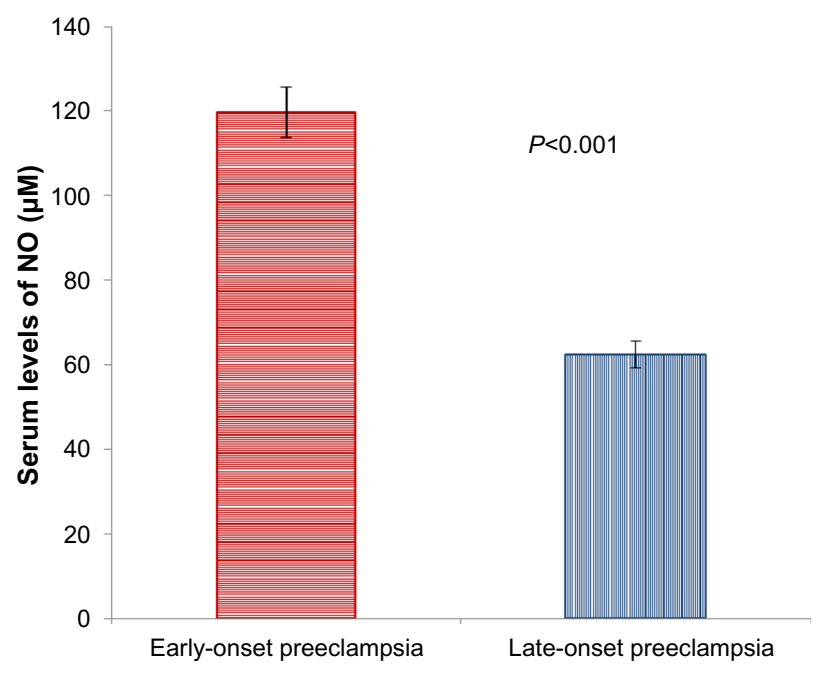

Figure 2 Mean serum levels of $\mathrm{NO}$ in early-onset and late-onset preeclampsia. Abbreviation: NO, nitric oxide.

\section{Discussion}

In this study, maternal serum NO levels were found to be significantly elevated in normotensive pregnant and in preeclamptic women compared to the nonpregnant state. The elevation in serum NO levels seen in PE was markedly greater than that seen in normal pregnancy, and this finding is consistent with other previous studies. ${ }^{9,14,15}$ Norris et al reported that the production of NO was significantly higher in the uteroplacental, feto-placental, and peripheral circulation in PE compared to normotensive pregnancies. They attributed the marked increase in NO levels to compensatory mechanism to the pathological effect of PE. ${ }^{14}$ Similarly, the marked elevation of NO levels in the PE determined in the current study might be attributable to compensatory reaction to restore the persistently elevated blood pressure.

Normal pregnancy is characterized by a profound increase in cardiac output and circulating intravascular volume. ${ }^{14}$ Blood pressure in normal pregnancy decreases from early pregnancy due to decreased peripheral resistance as a result of marked systemic vasodilatation. ${ }^{3} \mathrm{NO}$, a potent vasodilator, is thought to have a significant effect on gestational vasodilatation. Reduced NO synthase (NOS) activity resulting in decreased levels of NO may contribute significantly to the widespread vasoconstriction and hypertension in PE. ${ }^{14,16}$ However, NOS was not measured concomitantly with serum NO levels in this study. Although nitrate and nitrite levels were found to be markedly elevated in PE, it is possible that the amount of bioavailable NO needed to restore the elevated BP was insufficient.

However, a recent study has shown that constitutive endothelial NOS could be upregulated by a factor present in 
the sera of preeclamptic women. ${ }^{14}$ It has been demonstrated that endothelial cells exposed to $2 \%$ of plasma from preeclamptic women resulted in greater amounts of $\mathrm{NO}$ production and NOS activity compared to endothelial cells treated with plasma from normotensive uncomplicated pregnancies. ${ }^{17}$ This suggests that endothelial cell dysfunction due to certain substances released from the placenta plays a significant role in the pathogenesis of PE. Ranta et al also reported that NO production was increased in PE. ${ }^{18}$ Although the biologic significance of the increase is unknown, it might be a compensation for the generalized vasoconstriction occurring in PE. They stated that their result was unexpected, since PE is associated with marked vasoconstriction, which is generally considered as an evidence of decreased NO production. ${ }^{18}$ With the persistent hypertension, maternal serum levels of NO were expected to be reduced or deficient, but the converse was found in the current study.

Conversely, other researchers have indicated that maternal serum NO levels are reduced in PE. ${ }^{19}$ Sahu et al reported a reduction in NO production in preeclamptic women compared with normotensive pregnant women. ${ }^{19}$ In their study, they measured uric acid levels in plasma and urine concomitantly to address the possible impact of alteration in renal clearance on plasma NO metabolites in PE. In the current study, urinary uric acid levels were not measured concomitantly with the serum NO levels. However, the renal function of the participants was assessed using serum creatinine, urea, and uric acid levels concomitantly. Although there were significant differences in the mean serum creatinine, urea, and uric acid levels in the study population, the values were generally within normal limits indicating that renal function was not significantly impaired to affect NO clearance.

On the other hand, other researchers have reported that circulating nitrate and nitrite levels are not reduced in patients with severe PE compared with normotensive controls. ${ }^{20,21}$ Silver et al demonstrated that sera obtained from preeclamptic women did not suppress endothelial cell synthesis of NO in vitro as reported by other studies. ${ }^{20}$ The researchers evaluated the possibility that circulating factors in PE could suppress endothelial cells NO synthesis, but their study did not identify any significant suppressive effect on endothelial cell $\mathrm{NO}$ production in vitro. It was concluded that the absence of compensatory rise in NO synthesis in PE might also suggest a relative deficiency of $\mathrm{NO}$ due to impaired endothelial cell function. ${ }^{20}$

The current study also showed markedly elevated serum levels of NO in early-onset PE compared to late-onset PE that is considered as a mild disease. The significantly high levels of NO in early-onset PE might represent a compensatory mechanism to offset the pathologic effect of PE epitomized by generalized vasoconstriction and hypertension. ${ }^{14,18}$ The MAP was found to be significantly elevated in the preeclamptic group compared with the control groups (Table 1). Similarly, the mean arterial blood pressure was also significantly higher in early-onset disease compared to the late-onset PE. These findings might partly explain why the serum NO levels were also markedly elevated in the early-onset disease as a means of compensatory reaction to restore the ongoing pathological process. The markedly high serum levels of NO could still not restore the persistently elevated blood pressure, indicating a possible decoupling of signaling in endothelial cells or a consequence of generalized endothelial dysfunction. Intriguingly, there was no significant association between the MAP at diagnosis and maternal serum NO levels. This finding might be explained by the dysregulation of NO physiology in PE, probably from impaired endothelial function. However, significant negative correlation was found between the gestational age at diagnosis and NO levels suggesting that early-onset disease might be associated with marked endothelial damage.

Various analytical methods for quantitative measurement of nitrite and nitrate in plasma, serum, and urine of humans are available. ${ }^{22}$ In this study, concentration of nitrite and nitrate, which are stable products of the NO oxidation, was determined using Griess reagent. ${ }^{13}$ This assay measured the concentration of the serum nitrites and nitrates as a representation of total NO production. Griess reaction is simple and straightforward and has a long history with significant validity and reliability equivalent to contemporary methods. ${ }^{14}$

The limitation of this study includes the fact that determination of proteinuria was performed using a semiquantitative dipstick testing instead of a 24-hour urinary protein or urinary albumin/creatinine ratio, which is more accurate and reliable. Also, any inherent limitation of Griess reagent is considered a limitation of the current study. Despite the above limitations, the findings of this study serve as a baseline data for further longitudinal studies to better understand the contribution of $\mathrm{NO}$ alteration and endothelial dysfunction to the pathogenesis of PE.

\section{Conclusion}

In conclusion, the current study has determined significantly elevated NO levels in normal pregnancy, and more so, in $\mathrm{PE}$, compared to the nonpregnant state. The high levels of NO might be attributable to a dysregulated compensatory reaction to restore the generalized endothelial damage and 
persistently elevated hypertension characteristic of PE. The blood pressure elevation and serum NO levels were markedly higher in early-onset PE (severe disease) compared to the late-onset type (mild disease), suggesting a more severe endothelial dysfunction in the former. The findings from the current study and other previous studies show conflicting results in terms of NO production in PE. However, the baseline findings suggest that vascular endothelial cell dysfunction plays a pivotal role in the pathogenesis of PE.

\section{Acknowledgments}

The authors are grateful to the College of Health Sciences for funding this research work through the Bench Fee Award and a small grant for thesis and dissertations from the Association of African Universities. The authors are also grateful to the staffs of the Departments of Obstetrics and Gynecology, Physiology, and Biochemistry of the University of Ghana Medical School, labor wards of the KBTH, and the Noguchi Memorial Institute for Medical Research for facilitating the research. We also thank Mr Emmanuel Dwamena-Asare for his immense contribution to the data collection.

\section{Disclosure}

The authors report no conflicts of interest in this work.

\section{References}

1. Eiland E, Nzerue C, Faulkner M. Preeclampsia. J Pregnancy. 2012;586578:7.

2. Gilbert JS, Ryan MJ, LaMarca BB, Sedeek M, Murphy SR, Granger JP. Pathophysiology of hypertension during preeclampsia: linking placental ischemia with endothelial dysfunction. Am J Physiol Heart Circ Physiol. 2008;294(2):541-550.

3. Meher S, Duley L. Nitric oxide for preventing preeclampsia and its complications. Cochrane Database Syst Rev. 2007;18(2):CD006490.

4. Diejomaoh FME, Omu AE, Al-Busiri N, et al. Nitric oxide production is not altered in preeclampsia. Arch Gynecol Obstet. 2004;269: 237-243.

5. Adu-Bonsaffoh K, Oppong SA, Binlinla G, Obed SA. Maternal deaths attributable to hypertensive disorders in a tertiary hospital in Ghana. Int J Gynecol Obstet. 2013;123(2):110-113.
6. American College of Obstetricians and Gynecologists, ACOG. Diagnosis and management of preeclampsia and eclampsia. $A C O G$ Practice Bulletin. 2002;2:33.

7. Buhimschi IA, Saade GR, Garfield RE. The nitric oxide pathway in preeclampsia: pathophysiological implications (review). Hum Reprod Update. 1998;4:25-42.

8. Sladek SM, Magness RR, Conrad KP. Nitric oxide and pregnancy. Am J Physiol. 1997;272:R441-R463.

9. Shaamash AH, Elsnosy ED, Makhlouf AM, Zakhari MM, Ibrahim OA, Eldien HM. Maternal and fetal serum nitric oxide (NO) concentrations in normal pregnancy, preeclampsia and eclampsia. Int J Gynaecol Obstet. 2000;68:207-214.

10. Giustarini D, Rossi R, Milzani A, Dalle-Donne I. Nitrite and nitrate measurement by Griess reagent in human plasma: evaluation of interferences and standardization. Methods Enzymol. 2008;440:361-380.

11. George EM, Granger JP. Recent insights into the pathophysiology of preeclampsia. Expert Rev Obstet Gynecol. 2012;5(5):557-566.

12. Raymond D, Peterson E. A critical review of early-onset and late-onset preeclampsia. Obstet Gynecol Surv. 2011;66(8):497-506.

13. Granger DL, Taintor RR, Boockvar KS. Measurement of nitrate and nitrite in biological samples using nitrate reductase and Gries reaction. Methods Enzymol. 1996;268:142-151.

14. Norris AA, Higgins JR, Darling MRN, Walshe JJ, Bonnar J. Nitric oxide in the uteroplacental, fetoplacental, and peripheral circulations in preeclampsia. Am J Obstet Gynecol. 1999;93:958-963.

15. Nobunaga AK, Allman KG, Young D, Redman CWG. Elevated levels of serum nitrate, a stable end product of nitric oxide, in women with preeclampsia. Br J Obstet Gynaecol. 1997;104:538-543.

16. Choi JW, Im MW, Pai SH. Nitric oxide production increases during normal pregnancy and decreases in preeclampsia. Ann Clin Lab Sci. 2002;32(3):2002.

17. Davidge ST, Baker PN, Roberts JM. NOS expression is increased in endothelial cells exposed to plasma from women with preeclampsia. Am J Physiol. 1995;269:H1106-H1112.

18. Ranta V, Viinikka L, Halmesmaki E, Ylikorkala O. Nitric oxide production with preeclampsia. Obstet Gynecol. 1999;93:442-445.

19. Sahu S, Daniel M, Abraham R, Vedavalli R, Senthilvel V. Study of uric acid and nitric oxide concentrations in preeclampsia and normal pregnancy. Int J Biol Med Res. 2011;2(1):390-393.

20. Silver RK, Russel TL, Kupferminc MJ, Mullen TA, Caplan MS. Evaluation of nitric oxide as a mediator of severe preeclampsia. Am J Obstet Gynecol. 1996;175:1013-1017.

21. Davidge ST, Stranko CP, Roberts JM. Urine but not plasma nitric oxide metabolites are decreased in women with preeclampsia. Am J Obstet Gynecol. 1996;174:1008-1013.

22. Tsikas D. Methods of quantitative analysis of the nitric oxide metabolites nitrite and nitrate in human biological fluids. Free Radic Res. 2005;39:797-815.
Integrated Blood Pressure Control

\section{Publish your work in this journal}

Integrated Blood Pressure Control is an international, peer-reviewed open-access journal focusing on the integrated approach to managing hypertension and risk reduction. Treating the patient and comorbidities together with diet and lifestyle modification and optimizing healthcare resources through a multidisciplinary team approach constitute key

\section{Dovepress}

features of the journal. This journal is indexed on American Chemical Society's Chemical Abstracts Service (CAS). The manuscript management system is completely online and includes a very quick and fair peerreview system, which is all easy to use. Visit http://www.dovepress.com/ testimonials.php to read real quotes from published authors. 